\title{
REGULAR EMBEDDINGS OF MULTIGRAPHS
}

\author{
HUBERT DE FRAYSSEIX AND PATRICE OSSONA DE MENDEZ
}

\begin{abstract}
We prove that the vertex set of any twin-free loopless multigraph $G$ has an embedding into some point set $P$ of some Euclidean space $\mathbb{R}^{k}$, such that the automorphism group of $G$ is isomorphic to the isometry group of $\mathbb{R}^{k}$ globally preserving $P$.
\end{abstract}

\section{INTRODUCTION}

Using spectral analysis, Babai proved in 1978 that the abstract automorphism group of any multigraph $G$ having $s$ distinct eigenvalues with respective multiplicities $m_{1}, m_{2}, \ldots, m_{s}$ is a subgroup of $\boldsymbol{\omega}\left(m_{1}\right) \oplus \boldsymbol{\omega}\left(m_{2}\right) \oplus \cdots \oplus \boldsymbol{\omega}\left(m_{s}\right)$, where $\boldsymbol{\omega}(m)$ denotes the real orthogonal group of dimension $m$ [1]. As a consequence, if all the eigenvalues of $G$ are simple, the only automorphisms of $G$ are involutions.

Some years before, Mani proved that every triconnected planar graph $G$ can be realized as the 1-skeleton of a convex polytope $P$ in $\mathbb{R}^{3}$ such that all automorphisms of $G$ are induced by isometries of $P$ [7]. One non trivial consequence of this result is that the automorphism group $\operatorname{Aut}(G)$ of a triconnected planar graph $G$ has a chain of normal subgroups $\operatorname{Aut}(G)=G_{0} \triangleright G_{1} \triangleright \cdots \triangleright G_{m}=1$ where each quotient $G_{i} / G_{i-1}$ is either cyclic, or isomorphic to a symmetric group or $A_{5}$.

The result of Mani may be expressed in a weaker form: any triconnected planar graph has an embedding $f$ into $\mathbb{R}^{3}$, such that $\operatorname{Aut}(G)$ is the group of isometries of $\mathbb{R}^{3}$ globally preserving the point set $P=f(V(G))$, that we shall denote by $\boldsymbol{\omega}(3, P)$.

These two results suggest that a graph $G$ may possibly have some regular embedding, that is some embedding $f: V(G) \rightarrow \mathbb{R}^{k}$ such that Aut $(G)$ is isomorphic to the group $\boldsymbol{\omega}(k, f(V(G)))$ of isometries of $\mathbb{R}^{k}$ globally preserving $f(V(G))$, and that this group might be expressed as a subgroup of a group sum relying on spectral considerations. We shall prove that this is indeed the case for any twin-free loopless multigraph. In the remaining of this paper, multigraphs are always assumed to be loopless.

This result will be proved using techniques similar to the one used in a graph symmetry detection heuristic presented in GD'99 [4.

In section 2 we recall several definitions and introduce notations. In section 3 we reduce the study of the automorphism groups of multigraphs to the case of irreducible multigraphs, that is to multigraphs having no twin vertices. In section 4 we relate embeddings of a multigraphs to metrics and distance matrices on its vertex set. We reduce in section [5] the problem of finding regular embeddings to the one of finding metrics on the vertex set of the multigraph which define Euclidean, reconstructing and commuting distance matrices. We prove in section 6 that Euclidean distance matrices may be built from symmetric real matrices with

1991 Mathematics Subject Classification. Primary 05C50; Secondary 05 C62.

Key words and phrases. automorphism, distance, isometry, spectrum. 
0 on the diagonal, what we call predistance matrices. The built distance matrix is commuting/reconstructing if the predistance is. We deduce in section 7 that any commuting reconstructing predistance matrix defines a regular embedding and give some examples of reconstructing commuting predistance matrices. In section 8 we study the special case of regular multigraphs and give a strengthened version of Babai's result [1] in this context. Section 9 is devoted to concluding remarks.

\section{Definitions and Notations}

Let $G$ be a multigraph. For any $x, y \in V(G)$, the multiplicity $\mu(x, y)$ is the number of edges (possibly 0 ) having $x$ and $y$ as endpoints. An automorphism of $G$ is a one-to-one mapping $g: V(G) \rightarrow V(G)$ such that $\mu(g(x), g(y))=\mu(x, y)$ for any $x, y \in V(G)$. The automorphisms of $G$ define the automorphism group $\operatorname{Aut}(G)$ of $G$.

In order to ease the matrix presentation, we shall assume that the vertex set of a multigraph $G$ of order $n$ is $\{1, \ldots, n\}$. Then the adjacency matrix of $G$ is the symmetric matrix with entries $\boldsymbol{A}_{i, j}=\mu(i, j)$ and any automorphism $g \in \operatorname{Aut}(G)$ may be written as a permutation matrix $\boldsymbol{g}$, where $\boldsymbol{g}_{i, j}=\delta_{j, g(i)}(\delta$ is the usual Kronecker symbol). By permissive abridgment, $\operatorname{Aut}(G)$ would as well denote the group of these permutation matrices. Notice that $\operatorname{Aut}(G)$ may be then described as the group of the permutation matrices commuting with $\boldsymbol{A}$. Also, we will denote by $\boldsymbol{E}_{\boldsymbol{i}}$ the $n$ column matrix whose $j$ th entry is $\delta_{i, j}, \mathbf{1}$ the $n$ column matrix filled with 1 s and by $\boldsymbol{J}$ the $n \times n$ matrix $\mathbf{1 1}^{\mathrm{t}}$.

The symmetric group acting on a finite set $P$ will be denoted by $\mathfrak{S}(P)$. The real orthogonal group of dimension $n$ will be denoted by $\omega(n)$. If $P$ is a set of points of $\mathbb{R}^{n}$, the subgroup of $\omega(n)$ globally preserving $P$ will be denoted by $\omega(n, P)$. Notice that $\omega(n, P)<\mathfrak{S}(P)$.

\section{Reducing Graphs}

Definition 3.1. Let $G$ be a multigraph. $G$ is reducible if there exists two vertices $x_{1}, x_{2}$ such that $\mu\left(x_{1}, y\right)=\mu\left(x_{2}, y\right)$ for any $y \in V(G) \backslash\left\{x_{1}, x_{2}\right\}$. The vertices $x_{1}$ and $x_{2}$ are said to be twins. If a multigraph is twin-free, it is irreducible.

Lemma 3.1. Let $X$ be a subset of vertices, any two elements of which are twins. Then, $\mu(x, y)$ is constant for $x \neq y \in X$.

Proof. Let $X=\left\{x_{1}, x_{2}, \ldots, x_{k}\right\}$ Then, for $1<i<j \leq k: \mu\left(x_{i}, x_{j}\right)=\mu\left(x_{1}, x_{j}\right)$ as $x_{1}$ and $x_{i}$ are twins and $\mu\left(x_{1}, x_{j}\right)=\mu\left(x_{1}, x_{2}\right)$ as $x_{2}$ and $x_{j}$ are twins.

Lemma 3.2. Let $G$ be a multigraph. For any distinct vertices $x, y, z$ the following holds:

$$
\begin{array}{rll}
\text { If } x \text { and } y \text { are twins, that is: } & \forall v \in V(G) \backslash\{x, y\}, \quad \mu(x, v)=\mu(y, v) \\
\text { and } y \text { and } z \text { are twins, that is: } & \forall v \in V(G) \backslash\{y, z\}, \quad \mu(y, v)=\mu(z, v) \\
\text { then } x \text { and } z \text { are twins, that is: } & \forall v \in V(G) \backslash\{x, z\}, & \mu(x, v)=\mu(z, v) .
\end{array}
$$

Proof. Let $v \in V(G) \backslash\{x, z\}$.

If $v \neq y: \mu(x, v)=\mu(y, v)$ (as $x$ and $y$ are twins) and $\mu(y, v)=\mu(z, v)$ (as $y$ and $z$ are twins) hence $\mu(x, v)=\mu(z, v)$.

Otherwise $\mu(x, z)=\mu(x, y)$ (as $y$ and $z$ are twins) and $\mu(x, z)=\mu(y, z)$ (as $x$ and $y$ are twins) hence $\mu(x, v)=\mu(z, v)$. 
Corollary 3.3. The vertex set of any multigraph $G$ has a unique partition $\mathcal{P}=$ $\left\{V_{1}, \ldots, V_{k}\right\}$, such that any $V_{i}$ is a maximal subset of twin vertices of $G$. This partition is the twin-decomposition of $G$.

Corollary 3.4. Let $G$ be a multigraph, let $\mathcal{P}$ be its twin decomposition and let $G / \mathcal{P}$ denote the quotient multigraph. Then

$$
\operatorname{Aut}(G)=\bigoplus_{X \in \mathcal{P}} \mathfrak{S}(X) \oplus \operatorname{Aut}(G / \mathcal{P})
$$

Moreover, no subset $Y \subseteq V(G / \mathcal{P})$ of cardinality at least two is such that $\mathfrak{S}(Y)$ is a subgroup of $\operatorname{Aut}(G / \mathcal{P})$.

Proof. Assume there exists a subset $Y \subseteq V(G / \mathcal{P})$ of cardinality at least two is such that $\mathfrak{S}(Y)$ is a subgroup of $\operatorname{Aut}(G / \mathcal{P})$. Let $\tau$ be a transposition in $\mathfrak{S}(Y)$ exchanging two vertices $a$ and $b$ of $G / \mathcal{P}$. As $\tau$ is an automorphism of $G / \mathcal{P}$ it follows that $a$ and $b$ are twins in $G / \mathcal{P}$. Identifying $a$ and $b$ with the classes of twins of vertices in $G$, it follows that any $x \in a$ is a twin of any $y \in b$ in $G$ hence $a \cup b$ is a class of twins, what contradicts the maximality of the classes in $\mathcal{P}$.

\section{Embeddings And Distances}

Recall that a metric on a set $X$ is a mapping $\mathrm{d}: X^{2} \rightarrow \mathbb{R}^{+}$satisfying the usual axioms of a metric, that is:

$$
\begin{aligned}
\forall(x, y) & \in X^{2}, & & \mathrm{~d}(x, y)=\mathrm{d}(y, x) \\
\forall(x, y) & \in X^{2}, & & \mathrm{~d}(x, y)=0 \Longleftrightarrow x=y \\
\forall(x, y, z) & \in X^{3}, & & \mathrm{~d}(x, z) \leq \mathrm{d}(x, y)+\mathrm{d}(y, z)
\end{aligned}
$$

An Euclidean metric on a set $X$ is a metric d on $X$ such that there exist some Euclidean space $\mathbb{R}^{k}$ and an embedding $f: X \rightarrow \mathbb{R}^{k}$ so that, for any $x, y \in X$ :

$$
\operatorname{dist}(f(x), f(y))=\mathrm{d}(x, y)
$$

where dist is the Euclidean metric of $\mathbb{R}^{k}$. Any embedding of a multigraph $G$ into some Euclidean space $\mathbb{R}^{k}$ defines an Euclidean metric $\mathrm{d}_{f}$ on $V(G)$ by $\mathrm{d}_{f}(x, y)=$ $\operatorname{dist}(f(x), f(y))$.

Definition 4.1. The distance matrix of a metric $d$ on the set $\{1, \ldots, n\}$ is the $n \times n$ real symmetric matrix $\boldsymbol{D}$ defined by:

$$
\forall i, j \in\{1, \ldots, n\}, \quad \boldsymbol{D}_{i, j}=\mathrm{d}(i, j)^{2}
$$

Notice that the entries of $\boldsymbol{D}$ are the squares of the distances and not the distances themselves.

Definition 4.2. Let $G$ be a multigraph and let $\boldsymbol{D}$ be a distance matrix of a metric defined on $V(G)=\{1, \ldots, n\}$.

The distance matrix $\boldsymbol{D}$ is

- Euclidean if the metric from which $\boldsymbol{D}$ comes from is Euclidean. A compatible embedding of $G$ into an Euclidean space $\mathbb{R}^{k}$ is then called a $\boldsymbol{D}$-embedding of $G$;

- reconstructing if $G$ may be reconstructed from $\boldsymbol{D}$, that is:

$$
\exists \Xi: \mathbb{R} \rightarrow \mathbb{N}, \quad \forall i \neq j \in\{1, \ldots, n\}, \quad \mu(i, j)=\Xi\left(\boldsymbol{D}_{i, j}\right) ;
$$


- commuting if any automorphism of $G$ commutes with $\boldsymbol{D}$, that is:

$$
\forall g \in \operatorname{Aut}(G), \quad \boldsymbol{g} \boldsymbol{D}=\boldsymbol{D} \boldsymbol{g}
$$

or, equivalently:

$$
\forall g \in \operatorname{Aut}(G), \forall i, j \in\{1, \ldots, n\} \quad \boldsymbol{D}_{g(i), g(j)}=\boldsymbol{D}_{i, j} .
$$

\section{Distance Matrices and Regular Embeddings}

Definition 5.1. Let $G$ be a multigraph and let $k$ be an integer. A regular embedding of $G$ into $\mathbb{R}^{k}$ is a mapping $f: V(G) \rightarrow \mathbb{R}^{k}$ such that $\operatorname{Aut}(G)$ is isomorphic to $\omega(k, f(V(G)))$.

We recall the classical following theorem (which proof may be found in any undergraduate textbook):

Theorem 5.1 (Isometry Extension Theorem). Let $\left(a_{i}\right)_{i \in I}$ and $\left(b_{i}\right)_{i \in I}$ be families of points of an Euclidean affine space $\mathcal{E}$ (with distance dist) such that:

$$
\forall(i, j) \in I^{2} \quad \operatorname{dist}\left(a_{i}, a_{j}\right)=\operatorname{dist}\left(b_{i}, b_{j}\right)
$$

Then there exists an isometry $f$ of $\mathcal{E}$ such that:

$$
\forall i \in I \quad f\left(a_{i}\right)=b_{i}
$$

Moreover, if $\left(b_{i}\right)_{i \in I}$ spans $\mathcal{E}$ then $f$ is uniquely determined.

Theorem 5.2. Let $f$ be a one-to-one embedding of a multigraph $G$ into the Euclidean space $\mathbb{R}^{k}$ (with metric dist) and let $\boldsymbol{D}$ be the Euclidean distance matrix of the metric defined by $f$ on $V(G)$.

Assume $\boldsymbol{D}$ is reconstructing. Then $f$ is regular if and only if $f(V(G))$ spans $\mathbb{R}^{k}$ and $\boldsymbol{D}$ is commuting.

Proof. Assume $f(V(G))$ spans $\mathbb{R}^{k}$ and that $\boldsymbol{D}$ is commuting.

Let dist be the usual Euclidean metric of $\mathbb{R}^{k}$. Let $g \in \operatorname{Aut}(G)$. Then, for any vertices $x, y$ of $G$, $\operatorname{dist}(f(g(x)), f(g(y)))=\operatorname{dist}(f(x), f(y))$. According to Theorem $5.1 \mathrm{~g}$ may be extended to a unique isometry of $\mathbb{R}^{k}$ preserving $f(V(G))$, that is: to a unique element of $\omega(k, f(V(G))$.

Let $\tilde{g}$ denote the isometry defined by the automorphism $g$. Obviously, if $g_{1}, g_{2} \in$ $\operatorname{Aut}(G), \widetilde{g_{1} g_{2}}=\tilde{g_{1}} \widetilde{g_{2}}$. Thus we have defined a group morphism from $\operatorname{Aut}(G)$ to $\omega(k, f(V(G)))$.

Now assume $\phi \in \omega(k, f(V(G)))$. Define $g: V(G) \rightarrow V(G)$ by $f(g(x))=\phi(f(x))$. Notice that $g$ is well defined as $\phi$ is obviously one-to-one. As $\boldsymbol{D}$ is reconstructing, there exists a mapping $\Xi: \mathbb{R} \rightarrow \mathbb{N}$ such that $\Xi\left(\boldsymbol{D}_{i, j}\right)=\mu(i, j)$. Let $i \neq j \in$ $\{1, \ldots, n\}$. Then, for any $i \neq j \in\{1, \ldots, n\}$, we have: $\mu(i, j)=\Xi\left(\boldsymbol{D}_{i, j}\right)$. As $\phi$ is an isometry, the distance between $f(i)$ and $f(i)$ is the same as the distance as the distance between $\phi(f(i))$ and $\phi(f(i))$, that is: between $f(g(i))$ and $f(g(i))$ (by the definition of $g)$. It follows that $\boldsymbol{D}_{g(i), g(j)}=\boldsymbol{D}_{i, j}$ hence $\mu(g(i), g(j))=\mu(i, j)$. It follows that $g$ is an automorphism of $G$. Moreover, $\phi$ extends $f^{-1} \circ g \circ f$, thus $\phi=\tilde{g}$. It follows that $g \mapsto \tilde{g}$ is actually a group isomorphism from $\operatorname{Aut}(G)$ to $\omega(k, f(V(G)))$.

Conversely, assume $f$ is a regular embedding. Then $f(V(G))$ spans $\mathbb{R}^{k}$ for otherwise $\omega(k, f(V(G)))$ would not be a finite group although $\operatorname{Aut}(G)$ is. Let 
$\phi \in \omega(k, f(V(G)))$. Define $\tilde{\phi}: V(G) \rightarrow V(G)$ by $f(\tilde{\phi}(x))=\phi(f(x))$. As previously, we get $\mu(\tilde{\phi}(x), \tilde{\phi}(y))=m$, hence $\tilde{\phi}$ is an automorphism of $G$ such that $\mathrm{d}(f(\tilde{\phi}(x)), f(\tilde{\phi}(y)))=\mathrm{d}(f(x), f(y))$. Moreover, $\widetilde{\phi_{1} \phi_{2}}=\tilde{\phi_{1}} \tilde{\phi}_{2}$. It follows that $\phi \mapsto \tilde{\phi}$ is a group-morphism. As it is clearly one-to-one and as $\operatorname{Aut}(G)$ and $\omega(k, f(V(G)))$ are isomorphic by assumption, $\phi \mapsto \tilde{\phi}$ is onto. It follows that $\mathrm{d}(f(g(x)), f(g(y)))=\mathrm{d}(f(x), f(y))$ for any $g \in \operatorname{Aut}(G)$, that is: $\boldsymbol{D}$ is commuting.

We shall consider now this result from another point of view:

Corollary 5.3. Let $G$ be a multigraph and let $\boldsymbol{D}$ be an Euclidean reconstructing and commuting distance matrix on $G$. Then $\boldsymbol{D}$ defines a regular embedding of $G$.

Proof. Consider any $\boldsymbol{D}$-embedding $f$ of $G$ and the subspace spanned by $f(V(G))$ and apply Theorem 5.2

Our main problem is now to build such a distance matrix.

\section{Euclidean distance matrices from Predistance matrices}

Given a distance dist on the set $\{1, \ldots, n\}$, it is classical to define the corresponding bilinear form $\boldsymbol{B}$ by:

$$
\boldsymbol{B}=-\frac{1}{2}\left(\boldsymbol{I}-\frac{1}{n} \boldsymbol{J}\right) \boldsymbol{D}\left(\boldsymbol{I}-\frac{1}{n} \boldsymbol{J}\right)
$$

where $\boldsymbol{D}_{i, j}=\operatorname{dist}(i, j)^{2}$.

It is well known that the distance dist is Euclidean (i.e. allows an isometric embedding into some Euclidean space) if and only if $\boldsymbol{B}$ is positive semi-definite 2] 6]. Such a result extends to a characterization of those symmetric real matrices which are Euclidean distance matrices.

Definition 6.1. A predistance matrix on $G$ is an $n \times n$ symmetric real matrix $\boldsymbol{P}$ with 0 on the diagonal (notice that negative entries are allowed).

The bilinear form $\Lambda(\boldsymbol{P})$ of $\boldsymbol{P}$ is defined by:

$$
\Lambda(\boldsymbol{P})=-\frac{1}{2}\left(\boldsymbol{I}-\frac{1}{n} \boldsymbol{J}\right) \boldsymbol{P}\left(\boldsymbol{I}-\frac{1}{n} \boldsymbol{J}\right)
$$

Lemma 6.1. Let $\boldsymbol{D}$ be a predistance matrix and let $k$ be an integer. Then, the following statements are equivalent:

(1) $\boldsymbol{D}$ is an Euclidean distance matrix on $G$, and there exists a D-embedding of $G$ in $\mathbb{R}^{k}$;

(2) the matrix $\Lambda(\boldsymbol{D})$ is positive semi-definite and $\operatorname{rank}(\Lambda(\boldsymbol{D})) \leq k$.

Proof. Assume $\Lambda(\boldsymbol{D})$ is positive semi-definite. Then the square of the distance defined by $\Lambda(\boldsymbol{D})$ between the basis points $e_{i}$ and $e_{j}$ is $\left(\boldsymbol{E}_{i}-\boldsymbol{E}_{j}\right)^{t} \Lambda(\boldsymbol{D})\left(\boldsymbol{E}_{i}-\boldsymbol{E}_{j}\right)$. As $\left(\boldsymbol{I}-\frac{\boldsymbol{J}}{n}\right)\left(\boldsymbol{E}_{i}-\boldsymbol{E}_{j}\right)=\left(\boldsymbol{E}_{i}-\boldsymbol{E}_{j}\right)$, we get that the square of this distance equals $\left(\boldsymbol{D}_{i, j}+\boldsymbol{D}_{j, i}-\boldsymbol{D}_{i, i}-\boldsymbol{D}_{j, j}\right) / 2=\boldsymbol{D}_{i, j}$ as $\boldsymbol{D}$ is symmetric with zero on the diagonal. We define $f(i)$ as the projection of $e_{i}$ orthogonal to the isotropic space of $B$.

Conversely, assume $\boldsymbol{D}$ is an Euclidean distance matrix and let $f:[1 ; n] \rightarrow \mathbb{R}^{k}$ be a $\boldsymbol{D}$-embedding of $G$. Then $\Lambda(\boldsymbol{D})$ is the Gram matrix of the vectors $f(i)$, i.e. $\Lambda(\boldsymbol{D})_{i, j}=<f(i), f(j)>$. It is well known that this Gram matrix determines the vectors $f(i)$ up to isometry. 
Let $\boldsymbol{P}$ be a predistance matrix. The condition that $\Lambda(\boldsymbol{P})$ has no negative eigenvalue is quite difficult to handle. However, we know the following about $\Lambda(\boldsymbol{P})$ :

- $\Lambda(\boldsymbol{P})$ is symmetric real and thus diagonalizable,

- $\mathbf{1} \in \operatorname{ker}(\Lambda(\boldsymbol{P}))$ as $\left(\boldsymbol{I}-\frac{1}{n} \boldsymbol{J}\right) \mathbf{1}=\mathbf{0}$,

- $\Lambda(\boldsymbol{P})$ has an orthogonal basis $\mathcal{B}(\Lambda(\boldsymbol{P}))$ of eigenvectors including 1 . The eigenvectors of $\mathcal{B}(\Lambda(\boldsymbol{P})) \backslash\{\mathbf{1}\}$ have eigenvalues $\lambda_{1}>\cdots>\lambda_{r}$ with respective multiplicities $m_{1}, \ldots, m_{r}$.

Now consider the reduced distance matrix $\boldsymbol{P}^{\star}=\boldsymbol{P}-2 \lambda_{r}(\boldsymbol{J}-\boldsymbol{I})$.

Lemma 6.2. $\mathcal{B}(\Lambda(\boldsymbol{P}))$ is an orthogonal basis of eigenvectors of $\Lambda\left(\boldsymbol{P}^{\star}\right)$, and the eigenvalue (for $\Lambda\left(\boldsymbol{P}^{\star}\right)$ ) of $v \in \mathcal{B}(\Lambda(\boldsymbol{P}))$ is

$$
\begin{cases}0 & \text { if } \boldsymbol{v}=\mathbf{1} \\ \lambda-\lambda_{r} & \text { if } \boldsymbol{v} \neq \mathbf{1} \text { and } \Lambda(\boldsymbol{P}) \boldsymbol{v}=\lambda \boldsymbol{v}\end{cases}
$$

Thus, $\Lambda\left(\boldsymbol{P}^{\star}\right)$ is positive semi-definite and has corank $m_{r}+1$.

Proof. If $\boldsymbol{v} \neq \mathbf{1}$, then $\boldsymbol{v} \in \mathbf{1}^{\perp}$, and thus $\left(\boldsymbol{I}-\frac{\boldsymbol{J}}{n}\right) \boldsymbol{v}=\boldsymbol{v}$. Hence, if $\Lambda(\boldsymbol{P}) \boldsymbol{v}=\lambda \boldsymbol{v}$, then

$$
\begin{aligned}
\Lambda\left(\boldsymbol{P}^{\star}\right) \boldsymbol{v} & =-\frac{1}{2}\left(\boldsymbol{I}-\frac{\boldsymbol{J}}{n}\right) \boldsymbol{P}^{\star}\left(\boldsymbol{I}-\frac{\boldsymbol{J}}{n}\right) \boldsymbol{v} \\
& =-\frac{1}{2}\left(\boldsymbol{I}-\frac{\boldsymbol{J}}{n}\right)\left(\boldsymbol{P}-2 \lambda_{r}(\boldsymbol{J}-\boldsymbol{I})\right)\left(\boldsymbol{I}-\frac{\boldsymbol{J}}{n}\right) \boldsymbol{v} \\
& =-\frac{1}{2}\left(\boldsymbol{I}-\frac{\boldsymbol{J}}{n}\right) \boldsymbol{P}\left(\boldsymbol{I}-\frac{\boldsymbol{J}}{n}\right) \boldsymbol{v}+\lambda_{r}\left(\boldsymbol{I}-\frac{\boldsymbol{J}}{n}\right)(\boldsymbol{J}-\boldsymbol{I})\left(\boldsymbol{I}-\frac{\boldsymbol{J}}{n}\right) \boldsymbol{v} \quad \quad(\text { as } \boldsymbol{J} \boldsymbol{v}=\mathbf{0}) \\
& =\Lambda(\boldsymbol{P}) \boldsymbol{v}-\lambda_{r} \boldsymbol{v} \\
& =\left(\lambda-\lambda_{r}\right) \boldsymbol{v}
\end{aligned}
$$

\section{Reconstructing and Commuting predistance matrices}

Definition 7.1. A predistance matrix $\boldsymbol{P}$ is

- commuting if $\boldsymbol{P}$ commutes with any automorphism of $G$, that is:

$$
\forall g \in \operatorname{Aut}(G), \quad \boldsymbol{g} \boldsymbol{P}=\boldsymbol{P g}
$$

or, equivalently:

$$
\forall g \in \operatorname{Aut}(G), \forall i, j \in\{1, \ldots, n\}, \quad \boldsymbol{P}_{g(i), g(j)}=\boldsymbol{P}_{i, j} ;
$$

- reconstructing if there exists a mapping $\Xi: \mathbb{R} \rightarrow \mathbb{N}$ such that $\mu(i, j)=$ $\Xi\left(\boldsymbol{P}_{i, j}\right)$, or equivalently:

$$
\forall i, j, i^{\prime}, j^{\prime} \in\{1, \ldots, n\} \quad \boldsymbol{P}_{i, j}=\boldsymbol{P}_{i^{\prime}, j^{\prime}} \Rightarrow \mu(i, j)=\mu\left(i^{\prime}, j^{\prime}\right)
$$

Notation 7.2. Let $\boldsymbol{P}$ be a predistance matrix. As $\Lambda(\boldsymbol{P})$ has 0 as an eigenvalue, we may define $\zeta(\boldsymbol{P})$ as follows:

- if the smallest eigenvalue $\lambda$ of $\Lambda(\boldsymbol{P})$ is negative and has multiplicity $m$ then $\zeta(\boldsymbol{P})=n-m-1$,

- if the smallest eigenvalue of $\Lambda(\boldsymbol{P})$ is 0 with multiplicity $m>1$ then $\zeta(\boldsymbol{P})=$ $n-m$,

- if the smallest eigenvalue of $\Lambda(\boldsymbol{P})$ is 0 with multiplicity 1 and if the second smallest eigenvalue of $\Lambda(\boldsymbol{P})$ has multiplicity $m$ then $\zeta(\boldsymbol{P})=n-m-1$. 
Theorem 7.1. Let $G$ be an irreducible multigraph. Any commuting reconstructing predistance matrix $\boldsymbol{P}$ defines a regular embedding $f$ into $\mathbb{R}^{\zeta(\boldsymbol{P})}$.

Proof. Let $\boldsymbol{P}$ be a commuting reconstructing predistance matrix $\boldsymbol{P}$. The corresponding reduced distance matrix $\boldsymbol{P}^{\star}$, according to Lemma 6.1] and Lemma 6.2 is Euclidean and there exists a $\boldsymbol{P}^{\star}$ embedding $f$ on $G$ into $\mathbb{R}^{\zeta(\boldsymbol{P})}$. As $\boldsymbol{P}$ is reconstructing and commuting, so is $\boldsymbol{P}^{\star}$. Assume $f(x)=f(y)$. Since $f$ is reconstructing, $x$ and $y$ are twins of $G$, contradicting its irreducibility. Hence $f$ is one-to-one and, according to Theorem [5.2 $f$ is a regular embedding.

Corollary 7.2. Let $G$ be an irreducible multigraph and let $\boldsymbol{P}$ be a reconstructing predistance matrix which commutes with the automorphisms of $G$.

Denote by $1 \mathbb{R}$ the line spanned by $\mathbf{1}$ and by $\stackrel{\perp}{\oplus}$ the orthogonal direct sum of vector spaces. Let $\mathbf{1} \mathbb{R} \stackrel{\perp}{\oplus} E_{1} \stackrel{\perp}{\oplus} E_{2} \stackrel{\perp}{\oplus} \ldots \stackrel{\perp}{\oplus} E_{r}$ be a decomposition into eigenspaces of $\Lambda(\boldsymbol{P})$, the eigenvalues associated with $E_{1}, \ldots, E_{r}$ being $\lambda_{1}>\lambda_{2}>\cdots>\lambda_{r}$ (notice that some $\lambda_{i}$ may be 0$)$. Then the abstract automorphism group of $G$ is a subgroup of

$$
\boldsymbol{\omega}\left(\operatorname{dim} E_{1}, P_{1}\right) \oplus \boldsymbol{\omega}\left(\operatorname{dim} E_{2}, P_{2}\right) \oplus \cdots \oplus \boldsymbol{\omega}\left(\operatorname{dim} E_{r-1}, P_{r-1}\right)
$$

where $P_{i}$ is the orthogonal projection of the image of $G$ under the regular embedding defined by $\boldsymbol{P}$ into the subspace $E_{i}$.

Here some examples of simple commuting reconstructing predistance matrices of a simple connected graph $G$ (the two last examples only apply if $G$ has order at least 3):

$$
\begin{aligned}
& \text { Adjacency } \quad \boldsymbol{A}_{i, j}= \begin{cases}0, & \text { if } i=j \text { or } i \text { and } j \text { are adjacent } \\
1, & \text { otherwise }\end{cases} \\
& \text { Graph distance } \quad \boldsymbol{S}_{i, j}= \begin{cases}0, & \text { if } i=j \\
l, & \text { if } l \text { is the graph distance from } i \text { to } j\end{cases} \\
& \text { Bisected Czekanovski-Dice } \quad \boldsymbol{C}_{i, j}= \begin{cases}0, & \text { if } i=j \\
1-\frac{2}{d(i)+d(j)}, & \text { if } i \text { and } j \text { are adjacent } \\
1, & \text { otherwise }\end{cases} \\
& \text { Q-distance } \quad \boldsymbol{Q}_{i, j}= \begin{cases}0, & \text { if } i=j \\
1, & \text { if } i \text { and } j \text { are non adjacent } \\
1-\frac{1}{\sqrt{d(i) d(j)}}, & \text { otherwise }\end{cases}
\end{aligned}
$$

Theorem 7.3. Any irreducible multigraph has a regular embedding into some Euclidean space.

Proof. The adjacency matrix $\boldsymbol{A}$ of $G$ defined by $\boldsymbol{A}_{i, j}=\mu(i, j)$ is commuting and reconstructing. Hence the result follows from Theorem 7.1

\section{Regular Multigraphs}

As noted in section $\mathbf{7}$ the adjacency matrix $\boldsymbol{A}$ defines a commuting reconstructing predistance matrix. In the particular case where $G$ is $d$-regular, we have $\boldsymbol{A} \boldsymbol{J}=d \boldsymbol{J}$ thus $\Lambda(\boldsymbol{A})=(1-d / n)^{2} \boldsymbol{A}$. Moreover (see [3]): 
- $d$ is an eigenvalue of $G$ with eigenvector 1.

- If $G$ is connected, then the multiplicity of $d$ is one.

- For any eigenvalue $\lambda$ of $G$, we have $|\lambda| \leq d$.

In this case we deduce a strengthening of Babai's result [1] mentioned in section 1]

Corollary 8.1. Let $G$ be a connected irreducible regular multigraph having $s$ distinct eigenvalues $\lambda_{1}>\lambda_{2}>\cdots>\lambda_{s}$ with respective multiplicities $m_{1}, m_{2}, \ldots, m_{s}$. Then, the abstract automorphism group $\gamma$ of $G$ is a subgroup of

$$
\boldsymbol{\omega}\left(m_{2}, P_{2}\right) \oplus \boldsymbol{\omega}\left(m_{3}, P_{3}\right) \oplus \cdots \oplus \boldsymbol{\omega}\left(m_{s-1}, P_{s-1}\right) .
$$

Proof. Assume $G$ is connected. Let $\mathcal{B}(\boldsymbol{A})$ be an orthogonal basis of eigenvectors of $\boldsymbol{A}$. As $\boldsymbol{J} \boldsymbol{v}=0$ for any vector orthogonal to $\mathbf{1}$ and as $\boldsymbol{J} \mathbf{1}=n \mathbf{1}, \mathcal{B}(\boldsymbol{A})$ is also a basis of eigenvectors of $\Lambda(\boldsymbol{A})$. The eigenvalue (for $\Lambda(\boldsymbol{A})$ ) of an eigenvector $\boldsymbol{v}$ with eigenvalue $\lambda$ (for $\boldsymbol{A}$ ) is clearly $\lambda$ if $\lambda \neq d$ and 0 if $\lambda=d$. As the eigenvalue $d$ is maximal and simple for $\boldsymbol{A}$, we deduce that $\zeta(\boldsymbol{A})=n-m-1$, where $m$ is the multiplicity of the smallest eigenvalue of $\boldsymbol{A}$. Moreover, the greatest eigenvalue is simple and corresponds to eigenvector $\mathbf{1}$, according to Perron-Frobenius theorem [5] 8.

As a matter of fact, instead of $\boldsymbol{\omega}\left(m_{2}, P_{2}\right) \oplus \boldsymbol{\omega}\left(m_{3}, P_{3}\right) \oplus \cdots \oplus \boldsymbol{\omega}\left(m_{s-1}, P_{s-1}\right)$, we may prove a similar result with $\boldsymbol{\omega}\left(m_{3}, P_{3}\right) \oplus \cdots \oplus \boldsymbol{\omega}\left(m_{s-1}, P_{s-1}\right) \oplus \boldsymbol{\omega}\left(m_{s}, P_{s}\right)$ by considering $2 \lambda_{2}(\boldsymbol{J}-\boldsymbol{I})-\boldsymbol{A}$ instead of $\boldsymbol{A}-2 \lambda_{s}(\boldsymbol{J}-\boldsymbol{I})$.

A strongly regular graph is a regular graph such that there exist constants $\lambda, \mu$ so that any two adjacent vertices have exactly $\lambda$ common neighbors, and every two non-adjacent vertices have exactly $\mu$ common neighbors [9]. As a strongly regular graph has exactly three distinct eigenvalues [3], we may choose to keep among $E_{2}$ and $E_{3}$ the one having the smallest dimension. Hence every connected irreducible strong regular graph of order $n$ has a regular embedding into an Euclidean space of dimension at most $\left\lfloor\frac{n-1}{2}\right\rfloor$. As an example, the Petersen graph has a regular embedding into $\mathbb{R}^{4}$, which is optimal (the automorphism group of the Petersen graph cannot be realized as the isometry group of a set of points in $\mathbb{R}^{3}$ ).

\section{Conclusion}

The techniques presented here allow to construct regular embeddings from weakly constrained matrices: we only ask that they should be symmetric, have 0 on the diagonal, are reconstructing and commute with every automorphism.

Recall that a cellular algebra, or coherent algebra, is an algebra of $n \times n$ complex matrices which has a basis $\left\{\boldsymbol{B}_{0}, \boldsymbol{B}_{1}, \ldots, \boldsymbol{B}_{t}\right\}$ consisting of matrices with entries 0 and 1 satisfying the following conditions:

(1) $\boldsymbol{B}_{0}+\boldsymbol{B}_{1}+\cdots+\boldsymbol{B}_{t}=\boldsymbol{J}$

(2) $\exists 0 \leq r \leq t: \quad \boldsymbol{B}_{0}+\boldsymbol{B}_{1}+\cdots+\boldsymbol{B}_{r}=\boldsymbol{I}$;

(3) the set $\left\{\boldsymbol{B}_{0}, \boldsymbol{B}_{1}, \ldots, \boldsymbol{B}_{t}\right\}$ is closed under transposition.

The unique minimal cellular algebra which contains $\boldsymbol{A}$ as an element is the cellular algebra generated by $\boldsymbol{A}$ and is denoted by $<<\boldsymbol{A}\rangle>$. Notice that a basis of this cellular algebra may be constructed in polynomial time. In the case where $\boldsymbol{A}$ is the adjacency matrix of a simple graph $G$, the cellular algebra $\langle\langle\boldsymbol{A}\rangle\rangle$ is called the cellular algebra generated by $G$, or the cellular algebra of $G$. This algebra may contain non symmetric basis elements. However, any matrix in $\langle<A\rangle>$ commutes with any automorphism of $G$. It follows that any commuting predistance matrix $\boldsymbol{P}$ 
in $<<A>>$ may be written as $\sum_{i=r+1}^{t} \lambda_{i}\left(\boldsymbol{B}_{i}+\boldsymbol{B}_{i}^{\mathrm{t}}\right)$, with $\lambda_{i} \in \mathbb{R}$ for $r<i \leq t$ and thus form an affine space $\mathcal{E}$. By perturbing each of them (by adding $\epsilon(\boldsymbol{P}) \boldsymbol{A}$, for instance) we obtain reconstructing and commuting predistance matrices forming a dense subset of $\mathcal{E}$.

We hope that the suggested approach will be fruitful and more practical than the usual techniques arising from algebra and spectral analysis.

\section{REFERENCES}

[1] L. Babai, Automorphism group and category of cospectral graphs, Acta Math. Acad. Sci. Hung. 31 (1978), 195-306.

[2] J.P. Benzécri and et al, L'analyse des données - Tome II: l'analyse des correspondances, Dunod, Paris, 1973.

[3] N.L. Biggs, Algebraic graph theory, Cambridge tracts in mathematics, vol. 67, Cambridge University Press, 1974.

[4] H. de Fraysseix, An heuristic for graph symmetry detection, Graph Drawing (J. Kratochvíl, ed.), Lecture Notes in Computer Science, vol. 1731, Springer, 1999, pp. 276-285.

[5] G. Frobenius, Über Matrizen aus nicht negativen Elementen, S.-B. Preuss. Akad. Wiss. Berlin (1912), 456-477.

[6] S.L. Hakimi and S.S. Yau, Distance matrix of a graph and its realizability, Quaterly of Applied Mathematics 22 (1964), 305-317.

[7] P. Mani, Automorphismen von polyedrischen Graphen, Math. Ann. 192 (1971), 279-303.

[8] O. Perron, Zur Theorie der Matrizen, Math. Ann. 64 (1907), 248-263.

[9] J.J. Seidel, Strongly regular graphs, In Surveys in Combinatorics (Proc. Seventh British Combinatorial Conf., Cambridge, 1979) (Cambridge University Press, ed.), 1979, pp. 157-180.

Centre d'Analyse et de Mathématiques Sociales, CNRS, UMR 8557, 54 Bd Raspail, 75006 PARIS, France

E-mail address: hf@ehess.fr

Centre D'Analyse et de Mathématiques Sociales, CNRS, UMR 8557, 54 Bd Raspail, 75006 PARIS, France

E-mail address: pom@ehess.fr 